\title{
Adrenaline Content of Adrenals in Rabbits Poisoned with Acetylcholine and its Recovery
}

\author{
By \\ Susm Satoo \\ (伎藤 存) \\ (From the Physiological Laboratory of Prof. Y. Satake, \\ Tohoku University, Sendai) \\ (Received for publication, June 19, 1951) \\ The previous researches rê the production of adrenaline in the adrenal \\ gland were done to find whether the production is regulated by the nervous \\ control and then to determine its velocity. The agencies which act to \\ reduce the adrenaline content through the nervous route are: Diphtheria \\ toxine on rabbits, ${ }^{1 /}$ cold on cats, ${ }^{2)}$ physostigmine on dogs ${ }^{37}$ and rabbits, ${ }^{4)}$ etc. \\ In supplementing this series of researches, the acethylcholine was used \\ as a means for accelerating immensely the adrenaline discharge by acting \\ directly upon the adrenal medulla itself.5)
}

\section{Method:}

Rabbits, male and female without pregnancy, were used. The left splanchnicotomy was done without narcosis, and 21-68 days were then allowed to elapse until the experimentation. No food was given for 12 hours before the injection of the drug. Acetylcholine chloride (Roche) solution, prepared with saline solution, in $2.5 \%$ before the use, was hypodermically injected in a dose of 15 $\mathrm{mg}$. per $\mathrm{kg}$ body weight. At certain intervals as 5,12 \& 24 hours after the injection the animals were killed by a blow on the neck, and the adrenals were removed out soon. The Suto-Kojima's method was used for estimating the adrenaline.

The data are given in the accompanying table.

As the control 9 rabbits were used.

On injecting the drug the animals suffered therefrom; the severe symptoms lasted ten to thirty minutes. Afterwards they looked quite normal.

As the consequence the glands taken out 5 hours after the injection were estimated extraordinarily heavy, as both glands together $341 \mathrm{mg}$. in toto and $165 \mathrm{mg}$. per $\mathrm{kg} \mathrm{b} . \mathrm{w}$. as the average of 6 cases, against $211 \mathrm{mg}$. and $124 \mathrm{mg}$. per $\mathrm{kg}$. in the 9 control cases. The glands taken out 12 hours and 24 hours respectively after the injection did not show such an increase in weight. This affords also an evidence against the use of adrenaline 
TABLE

The Recovery of Adrenaline Content in the Denervated (Left) and in

\begin{tabular}{|c|c|c|c|c|c|c|}
\hline \multirow{2}{*}{ 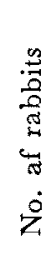 } & \multirow[b]{2}{*}{ Sex } & \multicolumn{3}{|c|}{ L. splanchnicotomy } & \multirow{2}{*}{ 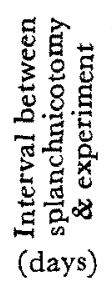 } & \multirow{2}{*}{$\begin{array}{l}\text { Experiment } \\
\text { Date } \\
(1941)\end{array}$} \\
\hline & & Date & $\begin{array}{l}\text { Body } \\
\text { weight } \\
\text { (kg.) }\end{array}$ & Route & & \\
\hline
\end{tabular}

Normal

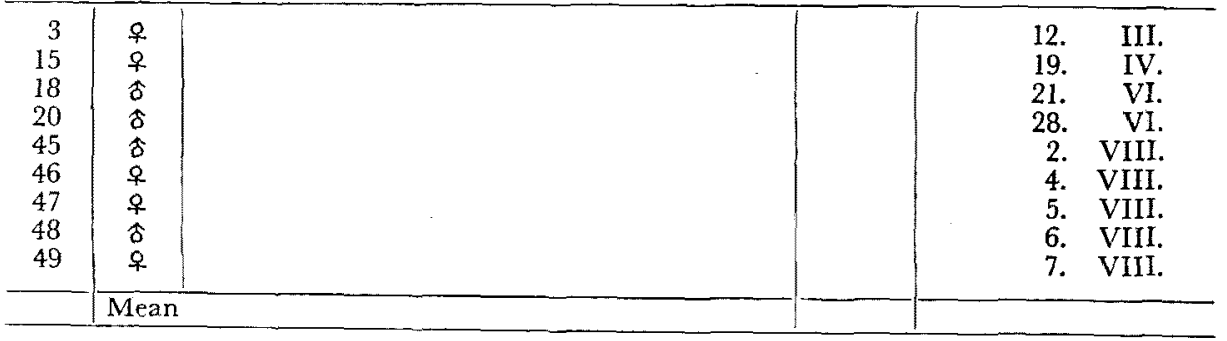

5 hours after the

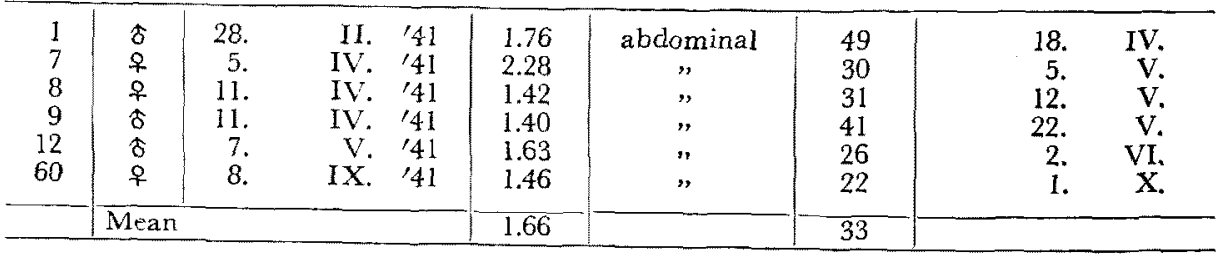

12 hours after the

\begin{tabular}{|c|c|c|c|c|c|c|c|c|}
\hline $\begin{array}{l}13 \\
16 \\
19 \\
36 \\
41 \\
59\end{array}$ & $\begin{array}{l}\hat{\delta} \\
\text { 今 } \\
0 \\
+ \\
\delta \\
0 \\
0 \\
0\end{array}$ & $\begin{array}{r}7 . \\
22 . \\
28 . \\
26 . \\
27 . \\
8 .\end{array}$ & $\begin{array}{l}\text { V. } \\
\text { V. } \\
\text { VI. } \\
\text { VII. } \\
\text { VII. } \\
\text { IX. }\end{array}$ & $\begin{array}{l}\prime 41 \\
\prime 41 \\
\prime 41 \\
41 \\
41 \\
41\end{array}$ & $\begin{array}{l}1.32 \\
1.59 \\
1.44 \\
2.07 \\
1.45 \\
1.34\end{array}$ & $\begin{array}{c}\text { abdominal } \\
", \\
", \\
\text { lumbar } \\
\text { abdominal }\end{array}$ & $\begin{array}{l}66 \\
58 \\
27 \\
25 \\
65 \\
21\end{array}$ & $\begin{array}{ll}13-14 . & \text { VII. } \\
19-20 . & \text { VII. } \\
25-26 . & \text { VII. } \\
20-21 . & \text { VIII. } \\
30 . & \text { IX. }-1 . X . \\
30 . & \text { IX. }-1 . X .\end{array}$ \\
\hline & \multicolumn{4}{|c|}{ Mlean } & 1.54 & & 44 & \\
\hline
\end{tabular}

24 hours after the

\begin{tabular}{|c|c|c|c|c|c|c|c|c|c|}
\hline $\begin{array}{l}22 \\
24 \\
25 \\
34 \\
50 \\
30\end{array}$ & $\begin{array}{l}q \\
+1 \\
0 \\
o \\
0 \\
q \\
q \\
q\end{array}$ & $\begin{array}{l}11 . \\
19 . \\
19 . \\
25 . \\
7 . \\
21 .\end{array}$ & $\begin{array}{l}\text { VII } \\
\text { VII } \\
\text { VII } \\
\text { VII } \\
\text { VIII } \\
\text { VII }\end{array}$ & $\begin{array}{l}141 \\
141 \\
141 \\
141 \\
141 \\
141\end{array}$ & $\begin{array}{l}2.00 \\
1.90 \\
1.60 \\
1.49 \\
2.08 \\
1.42\end{array}$ & $\begin{array}{c}\text { abdominal } \\
\text { ", } \\
\text { lumbar } \\
\text { abdominal } \\
\text { lumbar }\end{array}$ & $\begin{array}{l}24 \\
21 \\
21 \\
26 \\
21 \\
49\end{array}$ & $\begin{array}{c}4-5 . \\
9-10 . \\
9-10 . \\
20-21 . \\
28-29 . \\
8-9 .\end{array}$ & $\begin{array}{l}\text { VIII } \\
\text { VIII } \\
\text { VIII } \\
\text { VIII } \\
\text { VIII } \\
\text { IX. }\end{array}$ \\
\hline
\end{tabular}


I

the Innervated (Right) Glands after the Exhaustion by Acetylcholine

\begin{tabular}{|c|c|c|c|c|c|c|c|c|}
\hline \multirow{3}{*}{$\begin{array}{c}\text { Body } \\
\text { weight } \\
\text { (kg.) }\end{array}$} & \multicolumn{2}{|c|}{$\begin{array}{l}\text { Acetylcholine } \\
\text { injection }\end{array}$} & \multicolumn{6}{|c|}{ Weight of gland (mg.) } \\
\hline & \multirow{2}{*}{ 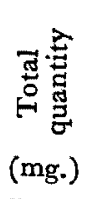 } & \multirow{2}{*}{ 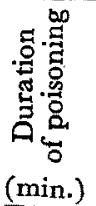 } & \multicolumn{2}{|c|}{ L } & \multicolumn{2}{|c|}{$\mathbf{R}$} & \multicolumn{2}{|r|}{$L-R$} \\
\hline & & & Total & $\begin{array}{l}\text { Per kg. } \\
\text { body } \\
\text { weight }\end{array}$ & Total & $\begin{array}{l}\text { Per kg. } \\
\text { body } \\
\text { weight }\end{array}$ & Total & $\begin{array}{c}\text { Per kg. } \\
\text { body } \\
\text { weight }\end{array}$ \\
\hline
\end{tabular}

control

\begin{tabular}{l|r|r|r|r|r|r|r}
\hline 1.36 & 97 & 71 & 111 & 82 & 208 & 153 \\
1.79 & 170 & 95 & 135 & 75 & 170 & 170 \\
2.10 & 106 & 50 & 104 & 50 & 100 & 100 \\
1.35 & 63 & 47 & 61 & 45 & 92 & 92 \\
1.97 & 117 & 59 & 104 & 53 & 112 & 112 \\
2.05 & 199 & 97 & 172 & 84 & 181 & 181 \\
1.35 & 97 & 72 & 79 & 59 & 130 & 130 \\
1.60 & & 68 & 43 & 71 & 44 & 87 & 87 \\
\hline 1.67 & & 44 & 44 & 72 & 43 & 87 & 87 \\
\hline
\end{tabular}

administration

\begin{tabular}{l|r|r|r|r|r|r|r|r|r|r|r|r|r|r|}
\hline 2.06 & 30.9 & 30 & 275 & 133 & 292 & 142 & 567 & 275 \\
2.43 & 36.5 & 27 & 397 & 163 & 317 & 130 & 714 & 294 \\
1.99 & 29.9 & 14 & 105 & 53 & 97 & 49 & 202 & 102 \\
1.96 & 29.4 & 18 & 72 & 37 & 67 & 34 & 139 & 71 \\
1.68 & 25.2 & 22 & 100 & 60 & 98 & 58 & 198 & 118 \\
1.70 & 25.5 & 10 & 113 & 66 & 111 & 65 & 224 & 132 \\
\hline 1.97 & 29.6 & 20 & 177 & 85 & 164 & 80 & 341 \\
\hline
\end{tabular}

administration

\begin{tabular}{l|l|l|l|l|l|l|l|l|}
\hline 2.38 & 35.7 & 40 & 120 & 50 & 108 & 45 & 228 & 96 \\
2.10 & 31.5 & 35 & 146 & 70 & 121 & 58 & 267 & 127 \\
1.65 & 24.8 & 15 & 94 & 57 & 88 & 53 & 182 & 110 \\
2.15 & 32.3 & 15 & 133 & 62 & 119 & 55 & 252 & 117 \\
1.85 & 27.8 & 21 & 105 & 57 & 99 & 54 & 204 & 110 \\
1.74 & 26.1 & 17 & 124 & 71 & 118 & 68 & 242 & 139 \\
\hline 1.98 & 29.7 & 24 & 120 & 61 & 109 & 56 & 229 & 117 \\
\hline
\end{tabular}

administration

\begin{tabular}{l|l|l|l|r|r|r|r|r|}
\hline 2.10 & 31.5 & 70 & 214 & 102 & 187 & 89 & 401 & 191 \\
2.06 & 30.9 & 15 & 104 & 50 & 94 & 46 & 198 & 96 \\
1.71 & 25.7 & 12 & 90 & 53 & 78 & 46 & 168 & 98 \\
1.45 & 22.0 & 14 & 60 & 41 & 50 & 34 & 110 & 76 \\
2.07 & 31.1 & 25 & 218 & 105 & 160 & 77 & 378 & 183 \\
1.61 & 24.2 & 14 & 132 & 82 & 124 & 77 & 256 & 159 \\
\hline 1.83 & 27.6 & 25 & 136 & 72 & 116 & 62 & 252 & 134 \\
\end{tabular}




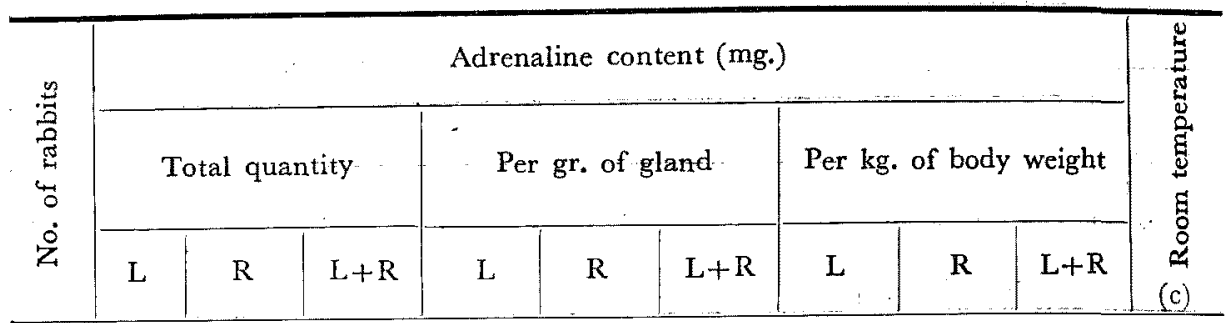

Normal control

\begin{tabular}{r|c|c|c|c|c|c|c|c|c|c}
\hline 3 & 0.078 & 0.080 & 0.158 & 0.80 & 0.72 & 0.76 & 0.057 & 0.059 & 0.116 & 11 \\
15 & 0.064 & 0.059 & 0.123 & 0.38 & 0.44 & 0.40 & 0.036 & 0.033 & 0.069 & 10 \\
18 & 0.084 & 0.080 & 0.164 & 0.79 & 0.77 & 0.78 & 0.040 & 0.038 & 0.078 & 20 \\
20 & 0.054 & 0.051 & 0.105 & 0.86 & 0.84 & 0.85 & 0.040 & 0.038 & 0.078 & 23 \\
45 & 0.080 & 0.080 & 0.160 & 0.68 & 0.77 & 0.72 & 0.041 & 0.041 & 0.081 & 22 \\
46 & 0.174 & 0.160 & 0.334 & 0.87 & 0.93 & 0.90 & 0.085 & 0.078 & 0.162 & 22 \\
47 & 0.058 & 0.048 & 0.106 & 0.60 & 0.61 & 0.60 & 0.043 & 0.036 & 0.078 & 27 \\
48 & 0.048 & 0.050 & 0.098 & 0.71 & 0.70 & 0.71 & 0.030 & 0.031 & 0.061 & 24 \\
49 & 0.070 & 0.070 & 0.140 & 0.95 & 0.97 & 0.96 & 0.042 & 0.042 & 0.084 & 27 \\
\hline & 0.079 & 0.075 & 0.154 & 0.74 & 0.75 & 0.74 & 0.046 & 0.044 & 0.090 & \\
\hline
\end{tabular}

5 hours after the administration

\begin{tabular}{r|r|l|l|l|l|l|l|l|l|l}
\hline 1 & 0.090 & 0.082 & 0.172 & 0.33 & 0.28 & 0.30 & 0.044 & 0.040 & 0.083 & 10 \\
7 & 0.068 & 0.072 & 0.140 & 0.17 & 0.23 & 0.20 & 0.028 & 0.030 & 0.058 & 17 \\
8 & 0.040 & 0.042 & 0.082 & 0.38 & 0.43 & 0.41 & 0.020 & 0.021 & 0.041 & 16 \\
9 & 0.044 & 0.044 & 0.088 & 0.61 & 0.66 & 0.63 & 0.022 & 0.022 & 0.045 & 19 \\
12 & 0.068 & 0.072 & 0.140 & 0.68 & 0.73 & 0.71 & 0.040 & 0.043 & 0.083 & 20 \\
60 & 0.052 & 0.058 & 0.110 & 0.46 & 0.52 & 0.49 & 0.031 & 0.034 & 0.065 & 18 \\
\hline & 0.060 & 0.062 & 0.122 & 0.44 & 0.48 & 0.46 & 0.031 & 0.032 & 0.063 & \\
\hline
\end{tabular}

12 hours after the administration

\begin{tabular}{l|l|l|l|l|l|l|l|l|l|l|l}
\hline 13 & 0.054 & 0.058 & 0.112 & 0.45 & 0.54 & 0.49 & 0.023 & 0.024 & 0.047 & 18 \\
16 & 0.045 & 0.041 & 0.086 & 0.31 & 0.34 & 0.32 & 0.021 & 0.020 & 0.041 & 23 \\
19 & 0.062 & 0.058 & 0.120 & 0.66 & 0.66 & 0.66 & 0.038 & 0.035 & 0.073 & 20 \\
36 & 0.080 & 0.082 & 0.162 & 0.60 & 0.69 & 0.64 & 0.037 & 0.038 & 0.075 & 25 \\
41 & 0.036 & 0.058 & 0.094 & 0.34 & 0.59 & 0.46 & 0.019 & 0.031 & 0.051 & 18 \\
59 & 0.058 & 0.044 & 0.102 & 0.47 & 0.37 & 0.42 & 0.033 & 0.025 & 0.059 & 18 \\
\hline & & & & & & & & & & & \\
\hline
\end{tabular}

24 hours after the administration

\begin{tabular}{l|l|l|l|l|l|l|l|l|l|l}
\hline 22 & 0.088 & 0.080 & 0.168 & 0.41 & 0.43 & 0.42 & 0.042 & 0.038 & 0.080 & 22 \\
24 & 0.042 & 0.040 & 0.082 & 0.40 & 0.43 & 0.41 & 0.020 & 0.019 & 0.040 & 19 \\
25 & 0.074 & 0.070 & 0.144 & 0.82 & 0.90 & 0.86 & 0.043 & 0.041 & 0.084 & 21 \\
34 & 0.058 & 0.060 & 0.118 & 0.97 & 1.20 & 1.07 & 0.040 & 0.041 & 0.081 & 24 \\
50 & 0.128 & 0.130 & 0.258 & 0.59 & 0.81 & 0.68 & 0.062 & 0.063 & 0.125 & 23 \\
30 & 0.052 & 0.070 & 0.122 & 0.39 & 0.56 & 0.48 & 0.032 & 0.043 & 0.076 & 25 \\
\hline
\end{tabular}


to the weight of gland as the adrenaline content in the case of rabbits. ${ }^{6}$ )

Then in consulting the figures of the total amount of adrenaline and the amount per $\mathrm{kg}$. of b.w. on the table, the general outcome will be extracted in the following paragraphs:

In all the series of experiments the amount of adrenaline in toto and per kg. b. w. as well was almost same in the fellow glands, i.e. in the left denervated and the right intactly innervated. The loss of innervation has no influence upon either the decrease of adrenaline store by acetylcholine or its recovery.

Secondary the adrenaline content was smaller in the series of 12 hours after the injection than in that of 5 hours. This proves that the gland is still losing adrenaline at that period. It is however obscure when namely before or after 12 hours after the injection the adrenaline load diminishes at most. Safely can it be said that the gland restores one fourth of the normal store of adrenaline in first 12 hours.

In short, the adrenaline store of adrenals in rabbits is reduced by acetylcholine, without regard to whether the innervation of glands interferred with or not. The drug is fully proved to attack the adrenal medulla directly as reported by previous investigators.

The gland is able to recover the adrenaline load at the rate of onefourth of the normal value in the second 12 hours after the injection of acetylcholine. The recovery process takes place independently of the innervation.

\section{References}

1) Mikami, Tohoku J. of Exp. Med., 1925, 6, 299.

2) Crowden, J. of Physiol., 1930, 68, 313.

3) Edmunds \& Smith, J. of Lab. \& Cl. Med., 1932, 17, 399.

4) Satow, Y., Tohoku J. of Exp. Med., 1938, 32, 257.

5) Inaba, E., ibid., 1935, 27, 245; Taneiti, ibid., 1940, 38, 147; Takita, will be published in this Journal; Wada, M. et al., the same.

6) Sato, H., Iwate Igaku Semmon Gakko Zassi, 1940, 4, 242. 